ARTÍCULO ORIGINAL

\title{
Cumplimiento, control de objetivos metabólicos, motivos de incumplimiento al tratamiento y a las recomendaciones prescriptas en adultos con diabetes tipo 2 en Argentina en 2015
}

María Amelia Linari ${ }^{1 *}$, Claudio González ${ }^{2}$, Guillermo Dieuzeide ${ }^{3}$, María Florencia-Badias ${ }^{4}$, María Inés Argerich $^{5}$, Mariela Echenique ${ }^{6}$, Gustavo Frechtel $^{7}$, Omar Álvaro ${ }^{6}$, María del Carmen Babus ${ }^{8}$, Diego Botta ${ }^{9}$, Maria Marta Curet $^{10}$, Susana Fuentes ${ }^{11}$, Sandra Geraci ${ }^{12}$, Silvia Lapertosa ${ }^{13}$, Astrid Libman ${ }^{14}$, Elizabeth Méndez ${ }^{15}$, Julieta Méndez ${ }^{16}$, María Florencia-Noguerol ${ }^{4}$, Verónica Ojeda ${ }^{17}$, Nelson Papini ${ }^{18}$, Víctor Previtera ${ }^{5}$, Erika Vago ${ }^{19}$, Carla Wassner ${ }^{20}$ y Débora Chan ${ }^{21}$

${ }^{1}$ Sección Endocrinología y Nutrición, Unión Obrera Metalúrgica (UOM), Vicente López, Provincia de Buenos Aires; ${ }^{2}$ Departamento de Farmacología de la Universidad Nacional de Buenos Aires, Ciudad Autónoma de Buenos Aires; ${ }^{3}$ Servicio del Hospital Nuestra Señora del Carmen de Chacabuco, Provincia de Buenos Aires; ${ }^{4}$ Hospital Provincial de Rosario, Santa Fe; ${ }^{5}$ Hospital Perrupato, San Martín, Mendoza; ${ }^{6}$ Centro Integral de Diabetes, Neuquén; ${ }^{7}$ Servicio de Nutrición, Hospital Sirio Libanés, Ciudad Autónoma de Buenos Aires; ${ }^{8}$ Centro Integral de Diabetes, Endocrinología y Nutrición (CIDEN), Jujuy; ${ }^{9}$ Hospital Bouquet, Neuquén; ${ }^{10} \mathrm{Hospital}$ Regional Dr. Ramón Carrillo, Santiago del Estero; ${ }^{11}$ Hospital El Cruce, Provincia de Buenos Aires; ${ }^{12}$ Servicio de Nutrición, Hospital Castex de San Martín, Provincia de Buenos Aires; ${ }^{13}$ Hospital Central J.R. Vidal, Corrientes, Argentina; ${ }^{14}$ Servicio de Endocrinología, Sanatorio Americano, Rosario, Santa Fe; ${ }^{15} \mathrm{Hospital} \mathrm{de} \mathrm{Agudos} \mathrm{Ramón} \mathrm{Madariaga,} \mathrm{Misiones;}{ }^{16} \mathrm{Hospital}$ Vélez Sarsfield, Ciudad Autónoma de Buenos Aires; ${ }^{17}$ Hospital Nacional de Clínicas, Córdoba; ${ }^{18}$ Área Departamental Luján de Cuyo, Mendoza; ${ }^{19}$ Área de diabetes,Centro Privado Regina, Río Negro; ${ }^{20}$ Servicio de Nutrición, Hospital Udaondo, Carapachay, Provincia de Buenos Aires; ${ }^{21}$ Universidad Tecnológica (UTN), Facultad Regional Buenos Aires, Ciudad Autónoma de Buenos Aires. Argentina

\section{RESUMEN}

Objetivo: Describir el grado de cumplimiento en los objetivos glucémicos según las características individuales y las recomendaciones dadas por el equipo de salud. Establecer los motivos de incumplimiento sobre el tratamiento prescripto. Metodología: Estudio transversal, que abarca ocho regiones geográficas en Argentina; se incluyeron 1,520 pacientes adultos con diabetes tipo 2. Se aplicó

\section{ABSTRACT}

Objective: To describe the degree of compliance in the glycemic objectives according to individual characteristics and to the recommendations given by the health team. Analyze the reasons for non-compliance with the prescribed treatment. Methodology: Crosssectional study. Out of 8 geographic regions in Argentina, 1520 adult patients with T2D were included. Authenticated validated ques-
Correspondencia:

* María Amelia Linari

E-mail: marimelinadyahoo.com
Fecha de recepción: 28-02-2019

Fecha de aceptación: 07-05-2019 DOI: 10.24875/ALAD.19000378 
cuestionario validado autorreferido con respuestas policotómicas de puntuación simple. Se analizaron variables sobre cumplimiento del tratamiento en general, la toma o aplicación de medicación, el monitoreo glucémico, la alimentación prescripta y los motivos de incumplimiento como: problemas económicos, problemas laborales, intolerancia a la medicación, olvido, incomodidad, miedo al pinchazo y cansancio. Se estudió el número y porcentual de respondedores para cada ítem. Resultados: El 38.16\% refirió incumplimiento al tratamiento en general; las causas mayores referidas fueron: por costos personales en el cuidado, por escaso conocimiento en diabetes y poca educación brindada. Conclusiones: Argentina se halla en similar rango de cumplimiento en comparación con otros países. Individualizar cualitativamente los motivos de incumplimiento permite vislumbrar causas estructurales como la falta de educación y los problemas económicos.

Palabras clave: Diabetes tipo 2. Adultos. Cumplimiento terapéutico. Adherencia al tratamiento. tionnaire with simple scoring polychotomic responses was applied. Variables were analyzed on compliance to treatment in general, the taking or application of medication, glycemic monitoring, prescribed food and the reasons for non-compliance such as: economic problems, work problems, intolerance to medication, forgetfulness, discomfort, fear of puncture, fatigue. The number and percentage of respondents for each item was studied. Results: $38.16 \%$ reported noncompliance with treatment in general; the major causes referred were personal costs in care, poor knowledge of diabetes and little education provided. Conclusions: Argentina is in a similar range of compliance compared to other countries. Qualitatively identifying the reasons for non-compliance allows us to glimpse structural causes such as lack of education and economic problems. (Rev ALAD. 2019;9:118-28)

Corresponding author: María Amelia Linari, marimelina@yahoo.com

Key words: Type 2 diabetes. Adults. Therapeutic compliance. Adherence to treatment.

\section{INTRODUCCIÓN}

Revisiones sobre estándares de gestión en el tratamiento y en la evaluación de la calidad de atención y adherencia en diabetes tipo 2 (DT2) informan sobre una atención subóptima la cual no cumple con las metas definidas y las recomendaciones vigentes $^{1,2}$. Conocer la magnitud y las limitaciones en la adherencia terapéutica y el cumplimiento terapéutico es necesario para la planificación e intervención de tratamientos efectivos. Una medición fiable del problema da soporte teórico para una posterior toma de decisiones, un cambio en las recomendaciones y en la evaluación sobre el estilo de comunicación, la participación de los pacientes y su núcleo familiar, y el propio equipo de salud, como también de otros proveedores de salud. Analizar conceptos que definen y caracterizan a la adherencia y al cumplimiento terapéutico, involucra también conocer sobre las conductas de adhesión y su incidencia en el ámbito de la salud del individuo ${ }^{3-5}$. Investigar sobre adherencia al tratamiento requiere aunar factores que participan en un proceso de responsabilidad compartida activa y voluntariamente entre el 
paciente y los profesionales de los servicios de salud ${ }^{6}$. Incluye la revalorización de variables cualitativas y subjetivas sobre una dimensión dinámica y multifactorial, fruto de un acuerdo entre el equipo médico y el paciente donde se intenta encontrar un equilibrio ${ }^{1}$. Adherencia se diferencia de compliance o cumplimiento en que esto último suele hacer referencia estricta a lo farmacoclínico (como el de tomar la medicación por parte del paciente tal cual le fuera prescripto por el médico tratante), limitándose meramente a las órdenes según le fueron dadas al paciente, con lo cual se le culpabiliza y refleja connotaciones negativas?

Es importante aclarar, dentro de las investigaciones sobre adherencia, que utilizar el término «cumplimiento terapéutico» como concepto suele ser necesario al momento del análisis cuantitativo. El cumplimiento terapéutico se define como el motivo por el cual el paciente considera, por autorreferencia, si efectúa o no la indicación o recomendación del profesional o equipo de salud. Incluye características como la toma de medicación para la diabetes como se ha recomendado, el cumplimiento del monitoreo glucémico recomendado, el cumplimiento con la dieta recomendada y los factores referidos al incumplimiento. En este estudio se propone conocer la dimensión del problema que representa el cumplimiento terapéutico a nivel nacional, a fin de colaborar con el desarrollo de intervenciones adaptadas a las características del grupo o región a las que estén dirigidas.

\section{OBJETIVOS}

Describir por autorreferencia el grado de cumplimiento terapéutico a las recomendaciones dadas por el equipo de salud y establecer los motivos de incumplimiento sobre el tratamiento en general, la medicación, el monitoreo glucémico y la alimentación prescripta en adultos con DT2 en Argentina durante el 2015.

Describir la muestra de pacientes adultos con DT2 según los antecedentes clínicos, características socioeconómicas, objetivos metabólicos y cumplimiento de objetivos metabólicos.

\section{METODOLOGÍA}

\section{Diseño}

Estudio transversal cuya población la constituyeron adultos con DT2 que se atendieron en diferentes centros de atención ubicados en Argentina.

\section{Muestra}

Se estimó la proporción de la población teniendo en cuenta resultados extraídos del metaanálisis de Toobert, et al. ${ }^{8}$ y del estudio de adherencia sobre una muestra representativa realizado en una obra social en $2013^{9}$ con adultos con DT2. Se estratificó en tres grupos según fueran jubilados, con o sin prestación de salud; se determinó un total a recluir de 1,560 pacientes sobre 8 regiones geográficas Argentina coincidentes a los capítulos de trabajo que divide la Sociedad Argentina de Diabetes. Sobre cada región se propuso reclutar en forma sistemática no menos de 65 pacientes con DT2 en cada lugar de atención (195 por zona geográfica), que incluyeron: adultos mayores de 65 años jubilados y adultos con y sin prestación en salud (prepaga o mutual u obra social) con 3 meses de diagnóstico de DT2 o más de antigüedad de la enfermedad, previo consentimiento informado firmado. No se incluyeron pacientes con disglucemia. Se invitó a todo paciente que cumpliera con criterios de inclusión en el periodo del primer semestre de 2015 hasta 
completar el número objetivo $(\mathrm{N})$. Las encuestas se respondieron en la sala de espera o en su domicilio para luego ser entregadas en la recepción del Servicio. Se respondieron 1,520 encuestas. El porcentual de no respuesta para el valor objetivo total correspondió a un $2.56 \%$.

\section{Instrumento de recolección}

Encuesta validada y adaptada que constó de dos partes:

- Parte 1: cuestionario rediseñado con datos demográficos, educacionales, socioeconómicos y clínicos de los pacientes (14 ítems autorreferidos de opciones múltiples con respuestas policotómicas de puntuación simple) ${ }^{10}$.

- Parte 2: Summary of Diabetes Self-Care Activities $(\operatorname{SDSCA})^{8}$.

\section{Variables consideradas}

- Aspectos personales: edad, sexo, presencia y tipo de prestación de salud (sin obra social, con obra social y jubilación), fecha de diagnóstico de DT2.

- Aspectos clínicos:

- Comorbilidades (antecedentes autorreferidos del paciente, que este refirió en alguna oportunidad al profesional de salud): hipertensión, presencia de eventos cardiovasculares, dislipidemia, insuficiencia hepática, trastornos en la coagulación o enfermedad crónica de los glóbulos rojos.

- Complicaciones crónicas (antecedentes autorreferidos del paciente, que este refirió en alguna oportunidad al profesional de salud): oculares, renales, neuropatías.

- Control metabólico: hemoglobina glucosilada (HbA1c), promedios de glucemias de los últimos tres meses según medidor de cada paciente (se consideraron los promedios de glucemia para el control metabólico en aquellos pacientes que referían complicaciones crónicas renales avanzadas, insuficiencia hepática y enfermedades hemáticas) y cumplimiento de los objetivos glucémicos. Se definieron si cumplía o no con los objetivos de control glucémico o metabólico en forma individualizada. Para esto, se categorizaron tres grupos según antecedentes y características, como lo refirieran los estándares de la Asociación Americana de Diabetes y la Asociación Europea para el Estudio de la Diabetes en $2012^{11}$ :

- Grupo 1: DT2 de reciente diagnóstico o menos de 10 años de conocido, sin antecedentes de hipoglucemia frecuente y/o grave, sin complicaciones crónicas de la enfermedad microvasculares o macrovasculares, HbA1c $<7 \%$ o promedios de glucemia $<150 \mathrm{mg} / \mathrm{dl}$.

- Grupo 2: DT2 con más de 10 años de antigüedad, antecedentes previos de hipoglucemia frecuente y/o grave, presencia de complicaciones microvasculares o macrovasculares, HbA1c entre 7 y $7.5 \%$ o promedios de glucemia entre 151 y $180 \mathrm{mg} / \mathrm{dl}$.

- Grupo 3: DT2 con historia de hipoglucemias severas (pérdida de conocimiento o necesidad de ayuda de un segundo para el tratamiento), hipoglucemias asintomáticas, expectativa de vida limitada, condiciones comórbidas extensivas, complicaciones avanzadas (retinopatía severa, insuficiencia renal crónica establecida), 
analfabeto (no escribe ni lee), que haya faltado en el último trimestre a su trabajo como consecuencia de una descompensación o complicación diabética, jubilación prematura a causa de la diabetes, HbA1c del 7.5-8.0\% o promedios de glucemia de $181-200 \mathrm{mg} / \mathrm{dl}$.

- Variables de adherencia. Se tuvieron en cuenta las dimensiones o factores incluidos por la Organización Mundial de la Salud $2004^{2}$ referente a lo socioeconómico, lo relacionado con el equipo o el sistema de salud, la asistencia sanitaria y la enfermedad propiamente dicha, (factores relacionados con el tratamiento y factores relacionados con el paciente). Se incluyó:

- Adherencia: operativamente se utilizó el cuestionario validado SDSCA (Toobert, Hampson y Glasgow, 20009).

- Cumplimiento terapéutico: motivo por el cual el paciente consideró, por autorreferencia, si efectuaba o no la indicación o recomendación del profesional de la salud. Incluyó características como toma de medicación para la diabetes, cómo se ha recomendado o si incumplió por problemas económicos, intolerancia a la medicación (efectos adversos), olvido o incomodidad; cumplimiento del monitoreo glucémico recomendado o si incumplió por problemas económicos, miedo al pinchazo, olvido o incomodidad; cumplimiento de la dieta recomendada o si incumplió por factores laborales, económicos, cansancio o incomodidad.

- Aspectos de calidad de vida: ocupación, ausentismo por DT2, jubilación prematura por DT2, reducción del sueldo consecuente a ausentismo por DT2, percepción de deterioro de calidad de vida por su DT2. Se diferenciaron los encuestados: mayores de 65 años de pensionados y dentro de los jubilados los que percibieron jubilación prematura por DT y los activos (aquellos que a pesar de estar jubilados aún trabajaban); los con prestación en salud y los sin prestación en salud.

- Aspectos socioeconómicos:

- Gasto de bolsillo del paciente: para cuantificarlo se estudió qué proporción abarcó el gasto en salud por mes, que incluyó el total en medicación para la diabetes exclusivamente más el total en tiras reactivas, el total en medicación ajena a la diabetes, el total en gasto de consultas al médico, estudios (análisis de sangre y orina, ecografías, estudios cardiológicos, etc.) con relación al ingreso del grupo familiar que convivía directamente con el paciente.

- Hogar de pertenencia: aquellos que convivían con el paciente encuestado y participaban en el consumo y la economía familiar.

- Ingreso per cápita: cociente entre el ingreso total del grupo familiar y la cantidad de convivientes.

- Ayuda económica o subsidio: presencia de subsidio otorgado por el estado o cualquier tipo de ayuda brindada ajena al grupo familiar conviviente.

- Jefe de hogar: conviviente que más dinero aporta en la economía del grupo familiar.

\section{Análisis estadístico}

Se confeccionó una ficha para recolección de los datos y luego una base de datos en Excel ${ }^{\circledR}$. Se procesaron los datos con InfoStat ${ }^{\circledR}$. Para las 
características a describir se utilizaron datos expresados como media, desviación estándar, mediana y porcentajes, se utilizaron los test de Kruskal-Wallis y de Mann-Whitney. Para evaluar el cumplimiento de las recomendaciones dadas por el equipo de salud y los motivos de incumplimiento, se estudió el número de respondedores para cada pregunta y el porcentual que refirieron cumplir la prescripción en forma regular o los motivos de incumplimiento.

\section{Aspectos éticos}

Se presentó el protocolo base aprobado. En los centros de reclutamiento que fue necesario, se aguardó autorización de la dirección médica de la institución correspondiente o de los comités de docencia e investigación. Se solicitó la firma del consentimiento informado en aquellos centros de reclutamiento en los cuales la institución lo exigió. Se realizó la encuesta. Se preservaron la confidencialidad de la información otorgada por los encuestados y los consentimientos.

\section{RESULTADOS}

En relación con las características de la muestra, de los 1.520 pacientes adultos con DT2 encuestados, 802 eran mujeres y 685 hombres ( 33 pacientes no revelaron su sexo), la media de edad fue de 60.2 años y la antigüedad de DT2 de 10.1 años. La media de HbA1C fue de $7.85 \%$ y las medias glucémicas de $150.34 \mathrm{mg} / \mathrm{dl}$. Respecto a las comorbilidades, el $60.74 \%$ de los encuestados eran hipertensos, el $13.89 \%$ presentaron eventos cardiovasculares y el $49.06 \%$ dislipidemia. En cuanto a la presencia de complicaciones crónicas de la enfermedad, autorreferidas en la encuesta, estas fueron: retinopatía en un $32.43 \%$, enfermedad renal crónica en un $9.85 \%$ y neuropatías en un $25.85 \%$ (Tablas 1, 2 y 3).
TABla 1. Descripción de la muestra $(n=1,520)$

\begin{tabular}{|l|c|c|}
\hline Región & $\mathbf{n}$ & $\%$ \\
\hline Buenos Aires & 261 & 17.17 \\
\hline CABA & 167 & 10.99 \\
\hline Comahue & 224 & 14.74 \\
\hline Centro & 67 & 4.41 \\
\hline Cuyo & 229 & 15.07 \\
\hline Litoral & 188 & 12.37 \\
\hline Noroeste & 140 & 9.21 \\
\hline Noreste & 244 & 16.05 \\
\hline
\end{tabular}

$\mathrm{n}$ : número de encuestados.

TABLA 2. Distribución de encuestados según cobertura en salud

\begin{tabular}{|l|c|c|}
\hline Cobertura & $\mathbf{n}$ & $\%$ \\
\hline Jubilado & 554 & 36.45 \\
\hline OS & 481 & 31.64 \\
\hline SOS & 485 & 31.91 \\
\hline
\end{tabular}

OS: con obra social; SOS: sin obra social; n: número de encuestados.

Al asociar el valor de HbA1c con presencia de complicaciones crónicas se encontraron diferencias estadísticamente muy significativas tanto en el grupo de los que presentaban comorbilidades de la enfermedad (test de Kruskal-Wallis $\chi^{2}, p=8.621 \mathrm{e}-07$ ) como los que no las presentaban ( $p=9.311 \mathrm{e}-15)$. Se observó mayor prevalencia de complicaciones crónicas en los individuos con más alta HbA1c y medias de glucemias de los últimos tres meses más elevadas.

Al analizar el cumplimiento de los objetivos metabólicos según características individuales y grupos según los criterios ADA/EASD 2012, la relación de los que cumplieron con los objetivos vs. los que incumplieron fue de $59.27 / 40.72 \%$. El mayor porcentual de alcance de cumplimiento de objetivos metabólicos correspondió al grupo 1, pacientes con DT2 de 
TABLA 3. Antecedentes según prestación de salud

\begin{tabular}{|c|c|c|c|}
\hline Variable & os & sos & Jubilados \\
\hline Sexo: fem/masc & $240 / 234$ & $256 / 218$ & $306 / 233$ \\
\hline Edad: X (DE) & $56.59(12.04)$ & $54.38(11.64)$ & $69.87(9.53)$ \\
\hline Antigüedad: DT2, en años & $8.95(6.82)$ & $8.85(7.6)$ & $12.64(10.5)$ \\
\hline \multicolumn{4}{|l|}{ Antecedentes: n (\%) } \\
\hline HTA & $223 / 257(53.54)$ & $187 / 297(61.36)$ & $181 / 373(67.32)$ \\
\hline EC & $426 / 55(11.43)$ & $420 / 65(13.30)$ & $460 / 94(16.96)$ \\
\hline DLP & $255 / 226(46.98)$ & $237 / 248(51.13)$ & $282 / 272(49.09)$ \\
\hline RTN & $371 / 110(22.86)$ & $285 / 200(41.23)$ & $370 / 184(33.21)$ \\
\hline ER & $461 / 20(4.15)$ & $416 / 67(13.87)$ & 490/64 (11.55) \\
\hline Neuropatía & $416 / 65(13.51)$ & $341 / 144(42.22)$ & $433 / 121(21.84)$ \\
\hline Alt. hepáticas & $445 / 36(7.84)$ & $429 / 56(13.05)$ & $482 / 72(14.93)$ \\
\hline $\mathrm{HbA} 1 \mathrm{c}$ & $7.45(1.56)$ & $8.22(4.36)$ & $7.88(1.46)$ \\
\hline Glucemias mg/dl: X (DE) & $140.88(49.8)$ & $164.98(63.49)$ & $145.17(42.91)$ \\
\hline Objetivos de control: cumpl./no cumpl: n (\%) & $260 / 150(63.41)$ & $197 / 196(50.12)$ & $299 / 166(64.30)$ \\
\hline \multicolumn{4}{|c|}{ Cumplimiento metabólico según grupos por objetivos de control: ADA/EASD ${ }^{11}: \mathrm{n}(\%)$} \\
\hline 1 & $167 / 64(61.67)$ & $103 / 72(58.85)$ & $141 / 45(75.80)$ \\
\hline 2 & $88 / 86(50.57)$ & $91 / 121(42.92)$ & $150 / 117(56.17)$ \\
\hline 3 & $5 / 0(100)$ & $3 / 6(33.33)$ & $8 / 4(66.66)$ \\
\hline
\end{tabular}

OS: obra social; SOS: sin obra social; fem: femenino; masc: masculino; DE: desviación estándar; HTA: hipertensión; EC: eventos cardiovasculares; DLP: dislipidemia; RTN: complicaciones oculares; ER: complicaciones renales; Alt. hepáticas: trastornos hepáticos severos; HbA1c: hemoglobina glucosilada; ADA/EASD: American Diabetes Association/European Association for the Study of Diabetes.

reciente diagnóstico o menos de 10 años de conocido.

Se analizaron variables de calidad de vida y socioeconómicas: refirió ausentismo a consecuencia de la DT2 un 10.68\% de los respondedores, reducción del sueldo por ausentismo consecuente a la DT2 un $10.45 \%$, jubilación prematura por DT2 un $27.91 \%$, percepción de pérdida de calidad de vida un $47.49 \%$ y percepción de subsidio o ayuda económica un $12.08 \%$.

La relación hombres/mujeres como jefes de familia fue 943/470 (107 de los encuestados no respondieron este ítem), donde la media de edad correspondió a 60.04 (desviación estándar [DE]: 13.38)/59.26 (DE: 13.17) años para hombres vs. mujeres.

El 9\% del gasto de bolsillo del núcleo familiar conviviente se asignaba a salud, dentro del cual un 52.9\% correspondía a gasto en tratamiento para la DT2.

Aplicando el test de Mann-Whitney se encontró asociación significativa entre los valores medianos del gasto en salud asignado por mes y el cumplimiento de objetivos metabólico ( $p<0.0001$ ). A mayor gasto en salud, mejor alcance de metas u objetivos, pero se debe diferenciar esta observación del impacto que ocasiona el mayor gasto de bolsillo en 
tratamiento para la DT2 (dos conceptos diferentes para tener en cuenta).

Respecto al cumplimiento de las recomendaciones dadas, al analizar por autorreferencia el grado de cumplimiento de las recomendaciones dadas por el equipo de salud y los motivos de incumplimiento sobre el tratamiento en general, la medicación, el monitoreo glucémico y la alimentación prescripta, se evaluó el número de respondedores para cada pregunta, el porcentual de respondedores que refirieron cumplir con la prescripción en forma regular y los motivos de incumplimiento, pudiendo responder a más de una opción descripta en la encuesta a la vez (Tabla 4). El 38.16\% refirió incumplimiento al tratamiento en general; las causas mayores referidas fueron: por costos personales en el cuidado un $15.69 \%$, por escaso conocimiento en diabetes un $15.6 \%$ y por poca educación brindada un $12.41 \%$. Los mayores motivos de incumplimiento de la toma o aplicación de medicación fueron: el 8.17\% por olvido, el $6.32 \%$ por problemas económicos y el 3.43\% por incomodidad. Sobre el incumplimiento al monitoreo glucémico, los mayores motivos fueron: el $8.24 \%$ por olvido, el $8.11 \%$ por temor y el $8.10 \%$ problemas económicos. Con relación al incumplimiento en la dieta: el $17.98 \%$ refirió cansancio, el $16.67 \%$ problemas económicos y el $14.62 \%$ incomodidad.

\section{DISCUSIÓN}

El estudio transversal Factores de Riesgo Asociados a la Diabetes y Cardiovasculares (FRADYC), que se realizó en Argentina entre diciembre de 2006 y marzo de 2007 e incluyó 1,858 pacientes ${ }^{12,13}$, encontró una $\mathrm{HbA} 1 \mathrm{c}$ media del $7.2 \%$ y en el estudio realizado en una obra social en la provincia de Buenos Aires en $2013^{9}$ la media de HbA1c fue del 7.94\%; en comparación con el multicéntrico, que recolectó datos durante el 2015, la media de HbA1c para todas las regiones
TABLA 4. Grado de cumplimiento sobre el tratamiento en general, la medicación, el monitoreo glucémico, la alimentación prescripta y motivos de incumplimiento de los encuestados con diabetes tipo 2 (DT2) en 2015

\begin{tabular}{|c|c|}
\hline $\begin{array}{l}\text { Porcentual general de incumplimiento en las } \\
\text { recomendaciones dadas } \\
(n=1,520) \\
(n=580)\end{array}$ & $38.16 \%$ \\
\hline \multicolumn{2}{|l|}{$\begin{array}{l}\text { Motivos de incumplimiento general por autorreferencia } \\
\text { (más de una opción posible) }\end{array}$} \\
\hline Tratamiento estricto: & $5 \%$ \\
\hline Impedimento en la atención en el lugar de atención: & $12.93 \%$ \\
\hline Falta de motivación: & $10.69 \%$ \\
\hline Poca educación brindada sobre DT2: & $12.41 \%$ \\
\hline Escaso conocimiento sobre DT2: & $15.69 \%$ \\
\hline Costos personales en el cuidado: & $15.6 \%$ \\
\hline \multicolumn{2}{|l|}{$\begin{array}{l}\text { Motivos de incumplimiento en la toma de medicación } \\
\text { (más de una opción posible) }\end{array}$} \\
\hline Problemas económicos: & $6.32 \%$ \\
\hline Intolerancia a la medic: & $1.84 \%$ \\
\hline Por olvido: & $8.17 \%$ \\
\hline Por incomodidad: & $3.43 \%$ \\
\hline \multicolumn{2}{|l|}{$\begin{array}{l}\text { Motivos de incumplimiento en el monitoreo glucémico } \\
\text { (más de una opción posible) }\end{array}$} \\
\hline Problemas económicos: & $8.1 \%$ \\
\hline Miedo al pinchazo: & $8.11 \%$ \\
\hline Por olvido: & $8.24 \%$ \\
\hline Por incomodidad: & $3.43 \%$ \\
\hline \multicolumn{2}{|l|}{$\begin{array}{l}\text { Motivos de incumplimiento en la dieta } \\
\text { (más de una opción posible) }\end{array}$} \\
\hline Por motivos laborales: & $9.35 \%$ \\
\hline Por motivos económicos: & $16.67 \%$ \\
\hline Por cansancio: & $17.98 \%$ \\
\hline Por incomodidad: & $14.62 \%$ \\
\hline
\end{tabular}

de Argentina fue del $7.85 \%$. Se observó que a pesar del correr de los años y ajeno a los números de individuos incluidos en los totales de las muestras estudiadas, las medias continúan siendo similares.

Con respecto al cumplimiento de objetivos metabólicos, se encontró en un estudio realizado por Laiteerapong, et al., con similares metas glucémicas según antecedentes personales, que el 31\% (intervalo de confianza del 95\% [IC 95\%]: 27-34\%) de la población adulta de los EE.UU. con diabetes habría alcanzado metas de $\mathrm{HbA}_{1 \mathrm{C}}<7.0 \%$, y que el 69\% (IC 95\%: $66-73 \%)$ tendrían metas de $\mathrm{HbA} 1 \mathrm{c}$ menos estrictas 
(para dicha categoría el 31\% no alcanzó metas) ${ }^{14}$. En Cartagena, con similares criterios de inclusión y análisis ${ }^{6}$, se observó que el $42 \%$ no alcanzó las metas para objetivos glucémicos propuestos. En el estudio The International Diabetes Mellitus Practice Study (IDMPS) en pacientes pertenecientes a 17 países (incluido Argentina), el 36\% de los pacientes con DT2 no lograron objetivos de control glucémico impuesto ${ }^{15}$. En el Quality of care of people with type 2 diabetes in eight european countries: findings from the Guideline Adherence to Enhance Care (GUIDANCE) study, se incluyeron datos de las historias clínicas de personas con DT2 utilizando un protocolo común, tanto en centros de atención primaria como en especializados en ocho países europeos, refiriendo un porcentual de incumplimiento glucémico para el $46.4 \%{ }^{16}$. En un estudio de vigilancia en el que se analizaron los datos de los adultos con diabetes por medio de encuestas autorreferidas del National Health and nutrition Examination Survey (NHANES) y del Behavioral Risk Factor Surveillance System (BRFSS) para el periodo 2003-2004, el 43.2\% incumplió y para el periodo 2007-2010, el 47.8\% incumplió con los objetivos glucémicos impuestos ${ }^{17}$. En el estudio de UOM, 2103, en la provincia de Buenos Aires, se observó un $48 \%$ de la población encuestada con control de objetivos metabólicos inadecuado 9 , en el multicéntrico de 2015 , un $40.72 \%$ de la muestra incumplió con los objetivos metabólicos según recomendaciones. Los resultados de estos estudios muestran que un $41.89 \%$ de la población estudiada incumple con objetivos y, por consiguiente, las metas vigentes.

Respecto al cumplimiento e incumplimiento de las recomendaciones prescriptas por el equipo de salud, el European study to evaluate quality of life and treatment satisfaction in patients with type 2 diabetes mellitus (PANORAMA), realizado en Europa ${ }^{18}$, con relación al grado de control glucémico y sus determinantes, demostró que los factores relacio- nados con el hecho de no alcanzar los objetivos fueron: el objetivo previsto más exigente, la mayor antigüedad de la enfermedad, la edad más joven, la escasa adherencia a la opinión del médico sobre el estilo de vida o sobre la toma de la medicación. En el UOM de $2013^{9}$ de los motivos de incumplimiento en el tratamiento prescripto, los mayores porcentuales se refirieron al tratamiento estricto, lo que podría interpretarse también como objetivos considerados más exigentes para los pacientes. En nuestro estudio, los motivos prevalentes fueron por costos personales en el cuidado y por escaso conocimiento en diabetes. Varias de estas variables cualitativas directa e indirectamente responden a limitaciones entre el paciente y el equipo de salud en la educación y la comunicación.

Un estudio sobre el cumplimiento en la toma de medicación en DT2 se realizó en cuatro cadenas de farmacias comunitarias localizadas en Seattle, EE.UU., desde abril de 2008 hasta octubre de 2009, y se observó una dificultad en la toma de la medicación en un $27.1 \%^{19}$. En el UOM de $2013^{9}$ se observó un $22 \%$ de incumplimiento autorreferido en la toma de medicación; dentro de los motivos de incumplimiento, el mayor porcentual correspondió al olvido. En el multicéntrico de 2015 los mayores motivos fueron similares.

En lo referido al cumplimiento en la dieta recomendada en el UOM de 2013, entre los motivos de incumplimiento el mayor porcentaje fue para el cansancio, y en menor proporción la incomodidad y los problemas económicos. En el multicéntrico 2015 los mayores motivos a similares variables de respuesta.

En un estudio transversal ${ }^{20}$ donde intervenían pacientes con DT2 y educadores en DT se observó que el conocer cómo funcionan los medicamentos y su efectividad al disminuir la glucosa en sangre, sumado a cómo manejar los efectos adversos, constitu- 
yeron los tres factores de motivación comúnmente identificados para mejorar el cumplimiento y la adherencia a la medicación. El conocimiento sobre la medicación y sobre el monitoreo glucémico fue de gran ayuda en la optimización de la adherencia. El estudio demostró que los pacientes reconocían la educación como un factor importante en la mejora de la adherencia; factores como el olvido y los problemas económicos son variables coincidentes, referidas en diferentes estudios aquí descritos.

Una de las posibles limitaciones de este estudio es que al ser de tipo transversal, la naturaleza causal de las asociaciones encontradas no se puede determinar. Los factores de confusión residuales no pueden ser completamente excluidos. Es posible la presencia de sesgo de selección al elegir el espacio muestral donde se desarrolló el estudio respecto a una muestra no del todo representativa de la población de estudio en determinadas regiones, por consiguiente los resultados son limitados al describir determinadas características en la población objeto. Por último, el cuestionario realizado interrogó sobre resultados de control metabólico correspondientes a los últimos tres meses de realizada la encuesta.

\section{CONCLUSIONES}

Conocer los factores que favorecerían la motivación, el cumplimiento en la indicación prescripta y las características de la medicación prescripta, podría influir positivamente en la adherencia al tratamiento. La interrupción de los tratamientos prescriptos sigue siendo un obstáculo importante para lograr objetivos del tratamiento en pacientes con DT2. Ayudar a la comunicación entre todos los proveedores de salud, que incluyen médicos, educadores, enfermeros y farmacéuticos, podría garantizar que los pacientes reciban el mejor apoyo posible.
La evidencia científica muestra que con el correr del tiempo, respecto de los años de evolución de la DT2, el entusiasmo inicial decae. Con el avance tecnológico, el paciente y su núcleo familiar deben contar con la motivación para realizar un tratamiento que exige dedicación permanente, pero que con frecuencia aumenta el riesgo de una reducción en la adherencia al tratamiento. Si, en cambio, la persona con diabetes encuentra motivación y compromiso con respecto al tratamiento de la enfermedad, esta intervendrá con un accionar más activo, más implicado, más informado y educado, posiblemente con una mejor respuesta al tratamiento y mejora en los marcadores metabólicos. El estudio realizado en Argentina, el cual reclutó datos de 2015, demostró que nuestro país se encuentra en similar rango de cumplimiento con respecto a la mayoría de estudios internacionales, con diferencias respecto de países de mayor situación de vulnerabilidad socioeconómica o estudios que no hayan individualizado objetivos metabólicos según características y antecedentes del paciente como se realizó en este caso. La necesidad de garantizar acceso a la atención, medicamentos e insumos requieren de modelos de atención multifactorial para pacientes crónicos con DT2.

\section{AGRADECIMIENTOS}

Parte del trabajo se realizó con un subsidio otorgado por la Sociedad Argentina de Diabetes. Intervinieron en el reclutamiento de pacientes las provincias de Buenos Aires, Ciudad Autónoma de Buenos Aires (CABA), Neuquén, Río Negro, Córdoba, Mendoza, Santa Fe, Misiones, Corrientes, Jujuy y Santiago del Estero.

Se agradece la colaboración y participación en el reclutamiento de Leticia Barrera, Natalia Blanco, Raúl David, Alejandra Dib, Daniel Dionisi, Natalia Laguarde y Luis Neira. 


\section{BIBLIOGRAFÍA}

1. Información farmacoterapéutica de la Comarca (INFAC). Adherencia al tratamiento farmacológico en patologías crónicas Internet]. Gobierno Vasco, Departamento de Salud, Boletín INFAC; 2011 (vol. 1). Disponible en: https://www.osakidetza.euskadi.eus/contenidos/ informacion/cevime_infac/es_cevime/adjuntos/INFAC_v19_n3.pdf

2. Adherencia a los tratamientos a largo plazo: Pruebas para la acción [Internet]. Pan American Health Organization; 2004 [Citado en 2015]. Disponible en: http://www1.paho.org/spanish/ad/dpc/nc/ adherencia-largo-plazo.pdf

3. Iglay K, Cartier SE, Rosen VM, Zarotsky V, Rajpathak SN, Radican L, et al. Meta-analysis of studies examining medication adherence, persistence, and discontinuation of oral antihyperglycemic agents in type 2 diabetes. Curr. Med Res Opin. 2015;31(7):1283-96.

4. Haruhisa F, Miki M. Impact of nonadherence on complication risks and healthcare costs in patients newly-diagnosed with diabetes. Diabetes Res Clin Pract. 2017;123:55-62.

5. Joshi R, Joshi D, Cheriyath P. Improving adherence and outcomes in diabetic patients. Patient Prefer Adherence. 2017;11:271-5.

6. Alayón A, Mosquera Vásquez M. Adherencia al tratamiento basado en comportamientos en pacientes diabéticos. Cartagena de Indias, Colombia. Rev. Salud Pública. 2008;10(5):777-87.

7. Linari MA, Dieuzeide G, Basbus M, Noguerol F, Badias F. Adherencia al tratamiento en diabetes mellitus tipo 2. En: Saad M, Gagliardino JJ. Incretinas. Diabetes mellitus tipo 2: Abordaje multifactorial y el rol de las incretinas en su fisiopatología y tratamiento. 2. ${ }^{a}$ Edición. Buenos Aires: CENEXA, UNLP; 2018. pp. 149-60.

8. Toobert D, Hampson S, Glasgow R. The summary of diabetes selfcare activities measure: Results from 7 studies and a revised scale. Diabetes Care. 2000;23(7):943-50.

9. Linari MA. Factores que se asocian a la adherencia al tratamiento en diabetes mellitus tipo 2 en adultos del Gran Buenos Aires pertenecientes a la Unión Obrera Metalúrgica seccional Vicente López durante el 2013 [Tesis de maestría en Internet]. [Buenos Aires] Universidad Nacional de Lanús; 2015. Recuperado a partir de: http: www.unla.edu.ar/index.php/servicios-de-la-biblioteca

10. Linari MA, González C, Alvariñas J. Desarrollo y validación de un cuestionario sobre adherencia al tratamiento y costo de bolsillo en pacientes adultos con Diabetes Mellitus tipo 2. Rev Soc Argent Diabetes. 2010;44(4):336-7.
11. Inzucchi SE, Bergenstal RM, Buse JB, Diamant M, Ferrannini E, Nauck M, et al.; American Diabetes Association (ADA); European Association for the Study of Diabetes (EASD) Management of hyperglycemia in type 2 diabetes: A patient centered approach. Position statement of the American Diabetes Association (ADA) and the European Association for the Study of Diabetes (EASD). Diabetes Care. 2012;35(6):1364-79.

12. López González E, Ruiz ML, Luongo AM, García AB, González CD, Burlando G, et al.; en nombre del Grupo FRADYC. Estudio multicéntrico de hipertensión arterial en pacientes con diabetes mellitus tipo 2 realizado por especialistas en Argentina. Rev. Asoc. Latinoam. Diabetes. 2011;19(2):29-36.

13. López González E, García AB, Ruiz ML, Luongo MA, González CD, Burlando G; Grupo Fradyc. Pacientes con diabetes mellitus tipo 2: ¿Cuántos presentaron valores lipídicos recomendados? Rev. Soc. Argent. Diabetes. 2012;4(6):9-13.

14. Laiteerapong N, John PM, Nathan AG, Huang ES. Public health implications of recommendations to individualize glycemic targets in adults with diabetes. Diabetes Care. 2013;36(1):84-9.

15. Chan J, Gagliardino JJ, Baik SH, Chantelot JM, Ferreira S, Hancu N, et al.; On behalf of the IDMPS. Multifaceted determinants for achieving glycemic control: The International Diabetes Management Practice Study (IDMPS). Diabetes Care. 2009;32(2):227-33.

16. Stone MA, Charpentier G, Doggen K, Kuss O, Lindblad U, Kellner C, et al.; GUIDANCE Study Group. Quality of care of people with type 2 diabetes in eight european countries: findings from the Guideline Adherence to Enhance Care (GUIDANCE) study. Diabetes Care. 2013; 36:2628-38.

17. Mohammed K, Ali K, McKeever B, Jinan B, Saaddine C, Cowie G, et al. Achievement of goals in U.S. Diabetes Care 1999-2010. N Engl J Med. 2013;368:1613-24.

18. Velasco P, Parhofer KG, Bradley C, Eschwège E, Gönder-Frederick L, Maheux $\mathrm{P}$, et al. Current level of glycaemic control and its associated factors in patients with type 2 diabetes across Europe: data from the PANORAMA study. Clin Endocrinol. 2012;80(1):47-56.

19. Odegard PS, Carpinito G, Christensen DB. Medication adherence program: Adherence challenges and interventions in type 2 diabetes. J Am Pharm Assoc (2003). 2013;53:267-72.

20. Morello CM, Chynoweth M, Kim H, Singh RF, Hirsch JD. Strategies to improve medication adherence reported by diabetes patients and caregivers: Results of a taking control of your diabetes survey. Ann Pharmacother. 2011;45(2):145-53. 